\title{
Proving termination by invariance relations
}

\author{
Paolo Pilozzi` and Danny De Schreye \\ Dept. of Computer Science, K.U.Leuven, Belgium \\ paolo.pilozzi@cs.kuleuven.be danny.deschreye@cs.kuleuven.be
}

\begin{abstract}
We propose a new constraint-based approach to termination analysis, applicable to Logic Programming (LP) and Constraint Handling Rules (CHR). Our approach further extends the existing constraintbased approaches for LP based on polynomial interpretations and introduces a whole new level of expressivity. We can handle problems such as bounded increase and integer arithmetic, elegantly. Furthermore, we are able to prove termination of programs that only terminate for subsets of the considered queries. Examples are algorithms that manipulate graphs and that only terminate if the graph in the input is cycle-free. This information cannot be represented, using the existing techniques in termination analysis. Therefore, we introduce invariance relations, representing relations among terms that hold on atoms during calls to the program. These relations can also be derived in a constraint-based manner and they can be used as a basis for a more expressive interpretation of the atoms of the program. We discuss our technique in the context of CHR, solving an important class of open problems containing transitivity rules. We also demonstrate the technique in an LP context and show that it is more powerful than existing constraint-based approaches.
\end{abstract}

The following CHR program [3, 7], computes the transitive closure of a graph.

$$
\text { transitivity@ } \operatorname{arc}(X, Y), \operatorname{arc}(Y, Z) \Rightarrow \operatorname{arc}(X, Z) .
$$

This program is representative for a large class of practical programs in CHR that cannot be handled using any existing automated technique. For it to be proven terminating, the query graph may not contain cycles. Consider for example a query of two constraints, $\operatorname{arc}(a, b)$ and $\operatorname{arc}(b, c)$, representing a cycle-free graph, consisting of three nodes: $a, b$ and $c$. The transitivity rule is applicable to these constraints, adding the constraint $\operatorname{arc}(a, c)$. Since a fire-once policy prevents multiple applications of a propagation rule on the same combination of constraints, the program terminates. If on the other hand, the query graph contains a cycle, then arcs are added progressively along that cycle, ad infinitum.

To tackle the problem of programs that only terminate for some subset of the considered queries, we present in the next paragraphs a new approach to constraint-based termination analysis. The technique is based on polynomial interpretations [5], however, we allow more expressive polynomials than the ones used in [5]. Our approach is applicable to both LP and CHR programs. First,

* Supported by I.W.T. Flanders - Belgium 
we discuss our approach for CHR programs containing a transitivity rule. Then, we show the technique's applicability to LP and argue that it is more powerful than existing approaches.

To prove termination automatically, we develop verifiable sufficient conditions $[2,6]$. To express such conditions, we require information on possible calls to a program. Such information comes in the form of a call set. In LP, this corresponds to the set of atoms, selected in some derivation of the program, for some query. In CHR, it corresponds to the atoms used to fire a rule in some computation of the program for some query. To specify the intended use of a program, we use a query set, finitely represented using query patterns. From it, we select atoms to compose queries. Then, by application of abstract interpretation, we derive from query patterns, call patterns $[4,7]$ as a representation of the call set.

In [5], termination is proven using a polynomial interpretation. There, the interpretation maps atoms and terms to $\mathbb{N}$-closed polynomials, using a polynomial level mapping, |.|, for atoms and a polynomial norm, $\|$.$\| , for terms. The call$ set has to be rigid w.r.t. this interpretation, meaning that only polynomials can be constructed using the instantiated parts of atoms and terms. To verify this, syntactic conditions are constructed, based on call patterns [1].

In [5], the $\mathbb{N}$-closed polynomials that are used, are constructed from positive monomials. For example, a polynomial $a_{0}+a_{1} \cdot X_{1}+\cdots+a_{n} \cdot X_{n}$. However, for programs such as our running example, we require negative monomials to prove termination. We allow therefore polynomials that are the difference of two (linear) positive polynomials: $a_{0}^{1}+a_{1}^{1} \cdot X_{1}+\cdots+a_{n}^{1} \cdot X_{n}-\left(a_{0}^{2}+a_{1}^{2} \cdot X_{1}+\cdots+a_{n}^{2} \cdot X_{n}\right)$. For such polynomials, $\mathbb{N}$-closedness is not trivial: there is obviously a positive integer assignment to variables and coefficients, resulting in a negative integer outcome. To guarantee that such polynomials are $\mathbb{N}$-closed, $a_{0}^{1}+a_{1}^{1} \cdot X_{1}+\cdots+a_{n}^{1} \cdot X_{n} \geq$ $a_{0}^{2}+a_{1}^{2} \cdot X_{1}+\cdots+a_{n}^{2} \cdot X_{n}$ must hold during the execution of the program. In the next paragraph, we derive this information, using invariance relations.

To prove termination of a CHR program containing a transitivity rule, we need to be able to represent more restricted kinds of queries. Call patterns [4] provide information on the instantiated parts of atoms in the call set, however, do not represent relations that hold among these parts. To represent this information, we introduce invariance relations, holding on atoms in the query or the call set.

To prove termination of our running example, we must guarantee the use of a cycle-free query graph. In such a graph, one can always find an ordering on nodes, such that every arc points from a strictly smaller sized node to a bigger sized node. Thus, the queries we can use are in $\left\{\bigwedge_{i=1, \ldots, n} \operatorname{arc}\left(t_{i}^{\prime}, t_{i}^{\prime \prime}\right) \mid \forall i: t_{i}^{\prime \prime} \succ t_{i}^{\prime}\right\}$.

Using an invariance relation $I_{\text {arc } / 2}^{\text {query }}=\left\{\left(t_{1}, t_{2}\right) \mid t_{1}, t_{2} \in\right.$ Term $_{P}, t_{\text {max }} \succ t_{2} \succ$ $\left.t_{1} \succ t_{\min }\right\}$, we can formulate cycle-freeness on the level of individual query atoms. The constants, $t_{\max }$ and $t_{\min }$, fix the domain of terms that can be used as nodes. The ordering on terms in arcs is expressed on individual atoms. In contrast to the invariant formulated on the query, the latter invariance relation therefore represents a relation holding on atoms that can be used in a query, while the former invariant represents actual queries. 
Given the invariance relation $I_{\text {arc/2 }}^{\text {query }}$ on query atoms, we aim to derive an invariance relation $I_{\text {arc } / 2}^{\text {callset }}$ on the atoms used in calls. For that purpose, we will now assume that $I_{\text {arc } / 2}^{\text {query }}$ is not just an invariance relation, but actually specifies the query atoms of interest. Since we also assume that the query atoms are a subset of the call atoms, the invariance relation $I_{a r c / 2}^{\text {callset }}$ on the call atoms must hold on the query atoms. Thus, $I_{\text {arc } / 2}^{\text {query }} \subseteq I_{\text {arc } / 2}^{\text {callset }}$. More explicitly, we get the condition $\forall t_{1}, t_{2} \in \operatorname{Term}_{P}:\left(t_{1}, t_{2}\right) \in I_{\text {arc } / 2}^{\text {query }} \rightarrow\left(t_{1}, t_{2}\right) \in I_{\text {arc } / 2}^{\text {callset }}$, meaning that the invariance relation on query atoms must be more restrictive than the one on atoms in the call set. On the basis of CHR rules, we derive rule conditions. For the transitivity rule, we get that $\forall t_{1}, t_{2}, t_{3} \in \operatorname{Term}_{P}:\left(t_{1}, t_{2}\right) \in I_{\text {arc } / 2}^{\text {callset }} \wedge\left(t_{2}, t_{3}\right) \in$ $I_{\text {arc } / 2}^{\text {callset }} \rightarrow\left(t_{1}, t_{3}\right) \in I_{\text {arc } / 2}^{\text {callset }}$, meaning that the invariance relation holding on $\operatorname{arc} / 2$ atoms, has to remain valid under transitivity.

We derive invariance relations on call atoms in a constraint-based manner, using a polynomial interpretation. That is, we reformulate the conditions by using a symbolic polynomial form for invariance relations in the same way as this is done for interargument relations in [5]. The invariance relation in polynomial form for the query is $I_{\text {arc } / 2}^{\text {query }}=\left\{\left(t_{1}, t_{2}\right) \mid\left\|t_{\max }\right\|>\left\|t_{2}\right\| \wedge\left\|t_{2}\right\|>\left\|t_{1}\right\| \wedge\left\|t_{1}\right\|>\left\|t_{\text {min }}\right\|\right\}$.

For the invariance relation on the atoms used in calls, we provide the same level of expressivity as for the query. Thus for the $\operatorname{arc} / 2$ constraints, we express their invariance relation using three symbolic polynomial inequalities: $I_{\text {arc } / 2}^{\text {callset }}=$ $\left\{\left(t_{1}, t_{2}\right) \mid i i_{0}^{1}+i i_{1}^{1} \cdot\left\|t_{1}\right\|+i i_{2}^{1} \cdot\left\|t_{2}\right\| \geq i o_{0}^{1}+i o_{1}^{1} \cdot\left\|t_{1}\right\|+i o_{2}^{1} \cdot\left\|t_{2}\right\| \wedge i i_{0}^{2}+i i_{1}^{2} \cdot\left\|t_{1}\right\|+\right.$ $i i_{2}^{2} \cdot\left\|t_{2}\right\| \geq i o_{0}^{2}+i o_{1}^{2} \cdot\left\|t_{1}\right\|+i o_{2}^{2} \cdot\left\|t_{2}\right\| \wedge i i_{0}^{3}+i i_{1}^{3} \cdot\left\|t_{1}\right\|+i i_{2}^{3} \cdot\left\|t_{2}\right\| \geq i o_{0}^{3}+i o_{1}^{3} \cdot\left\|t_{1}\right\|+$ $\left.i o_{2}^{3} .\left\|t_{2}\right\|\right\}$. To find values for the symbolic coefficients, we formulate the invariance conditions in terms of these symbolic polynomials. Thus, $\forall t_{1}, t_{2} \in \operatorname{Term}_{P}$ :

$\left\|t_{\text {max }}\right\|>\left\|t_{2}\right\| \wedge\left\|t_{2}\right\|>\left\|t_{1}\right\| \wedge\left\|t_{1}\right\|>\left\|t_{\text {min }}\right\| \rightarrow i i_{0}^{1}+i i_{1}^{1} \cdot\left\|t_{1}\right\|+i i_{2}^{1} \cdot\left\|t_{2}\right\| \geq i o_{0}^{1}+i o_{1}^{1} \cdot\left\|t_{1}\right\|+i o_{2}^{1} \cdot\left\|t_{2}\right\| \wedge$ $i i_{0}^{2}+i i_{1}^{2} \cdot\left\|t_{1}\right\|+i i_{2}^{2} \cdot\left\|t_{2}\right\| \geq i o_{0}^{2}+i o_{1}^{2} \cdot\left\|t_{1}\right\|+i o_{2}^{2} \cdot\left\|t_{2}\right\| \wedge i i_{0}^{3}+i i_{1}^{3} \cdot\left\|t_{1}\right\|+i i_{2}^{3} \cdot\left\|t_{2}\right\| \geq i o_{0}^{3}+i o_{1}^{3} \cdot\left\|t_{1}\right\|+i o_{2}^{3} \cdot\left\|t_{2}\right\|$

For the condition related to the transitivity rule, we get that $\forall t_{1}, t_{2}, t_{3} \in \operatorname{Term}_{P}$ :

$$
\begin{gathered}
i i_{0}^{1}+i i_{1}^{1} \cdot\left\|t_{1}\right\|+i i_{2}^{1} \cdot\left\|t_{2}\right\| \geq i o_{0}^{1}+i o_{1}^{1} \cdot\left\|t_{1}\right\|+i o_{2}^{1} \cdot\left\|t_{2}\right\| \wedge i i_{0}^{2}+i i_{1}^{2} \cdot\left\|t_{1}\right\|+i i_{2}^{2} \cdot\left\|t_{2}\right\| \geq \\
i o_{0}^{2}+i o_{1}^{2} \cdot\left\|t_{1}\right\|+i o_{2}^{2} \cdot\left\|t_{2}\right\| \wedge i i_{0}^{3}+i i_{1}^{3} \cdot\left\|t_{1}\right\|+i i_{2}^{3} \cdot\left\|t_{2}\right\| \geq i o_{0}^{3}+i o_{1}^{3} \cdot\left\|t_{1}\right\|+i o_{2}^{3} \cdot\left\|t_{2}\right\| \wedge i i_{0}^{1}+i i_{1}^{1} \cdot\left\|t_{2}\right\|+i i_{2}^{1} \cdot\left\|t_{3}\right\| \geq \\
i o_{0}^{1}+i o_{1}^{1} \cdot\left\|t_{2}\right\|+i o_{2}^{1} \cdot\left\|t_{3}\right\| \wedge i i_{0}^{2}+i i_{1}^{2} \cdot\left\|t_{2}\right\|+i i_{2}^{2} \cdot\left\|t_{3}\right\| \geq i o_{0}^{2}+i o_{1}^{2} \cdot\left\|t_{2}\right\|+i o_{2}^{2} \cdot\left\|t_{3}\right\| \wedge i i_{0}^{3}+i i_{1}^{3} \cdot\left\|t_{2}\right\|+i i_{2}^{3} \cdot\left\|t_{3}\right\| \geq \\
i o_{0}^{3}+i o_{1}^{3} \cdot\left\|t_{2}\right\|+i o_{2}^{3} \cdot\left\|t_{3}\right\| \rightarrow i i_{0}^{1}+i i_{1}^{1} \cdot\left\|t_{1}\right\|+i i_{2}^{1} \cdot\left\|t_{3}\right\| \geq i o_{0}^{1}+i o_{1}^{1} \cdot\left\|t_{1}\right\|+i o_{2}^{1} \cdot\left\|t_{3}\right\| \wedge i i_{0}^{2}+i i_{1}^{2} \cdot\left\|t_{1}\right\|+ \\
i i_{2}^{2} \cdot\left\|t_{3}\right\| \geq i o_{0}^{2}+i o_{1}^{2} \cdot\left\|t_{1}\right\|+i o_{2}^{2} \cdot\left\|t_{3}\right\| \wedge i i_{0}^{3}+i i i_{1}^{3} \cdot\left\|t_{1}\right\|+i i_{2}^{3} \cdot\left\|t_{3}\right\| \geq i o_{0}^{3}+i o_{1}^{3} \cdot\left\|t_{1}\right\|+i o_{2}^{3} \cdot\left\|t_{3}\right\|
\end{gathered}
$$

These conditions are similar to the ones obtained in [5] and thus can be solved in a similar way. That is, by transforming them to a system of Diophantine constraints on the symbolic coefficients and solving the resulting system by an existing constraint solver. One possible solution, results in an invariance relation,

$$
\begin{gathered}
I_{\text {arc }}^{\text {callset }}=\left\{\left(t_{1}, t_{2}\right) \mid\left\|t_{\max }\right\|+0 .\left\|t_{1}\right\|+0 .\left\|t_{2}\right\| \geq 0+0 .\left\|t_{1}\right\|+1 .\left\|t_{2}\right\| \wedge 0+0 .\left\|t_{1}\right\|+\right. \\
\left.1 .\left\|t_{2}\right\| \geq 0+1 .\left\|t_{1}\right\|+0 .\left\|t_{2}\right\| \wedge 0+1 .\left\|t_{1}\right\|+0 .\left\|t_{2}\right\| \geq\left\|t_{\text {min }}\right\|+0 .\left\|t_{1}\right\|+0 .\left\|t_{2}\right\|\right\}
\end{gathered}
$$

Thus, the invariance relation on the query is preserved under transitivity. That is, the bounds, $t_{\max }$ and $t_{\min }$, remain to be bounds for the added arcs and these arcs keep pointing from strictly smaller sized nodes to bigger sized nodes. 
To prove the transitivity program terminating, we obtain the following termination conditions, according to [6]. The scope of the decrease conditions is restricted by invariance relations holding on the atoms used in the head.

$$
\begin{aligned}
& \forall t_{1}, t_{2}, t_{3}:\left(t_{1}, t_{2}\right) \in I_{\text {arc } / 2}^{\text {callset }} \wedge\left(t_{2}, t_{3}\right) \in I_{\text {arc/2 }}^{\text {callset }} \rightarrow \operatorname{arc}\left(t_{1}, t_{2}\right) \succ \operatorname{arc}\left(t_{1}, t_{3}\right) \\
& \forall t_{1}, t_{2}, t_{3}:\left(t_{1}, t_{2}\right) \in I_{\text {arc } / 2}^{\text {callset }} \wedge\left(t_{2}, t_{3}\right) \in I_{\text {arc } / 2}^{\text {callset }} \rightarrow \operatorname{arc}\left(t_{2}, t_{3}\right) \succ \operatorname{arc}\left(t_{1}, t_{3}\right)
\end{aligned}
$$

To prove validity of these conditions, we require an interpretation, mapping $\operatorname{arc} / 2$ atoms to a polynomial of the form $\left|\operatorname{arc}\left(t_{1}, t_{2}\right)\right|=\left(\left\|t_{\max }\right\|-\left\|t_{\min }\right\|\right)-$ $\left(\left\|t_{2}\right\|-\left\|t_{1}\right\|\right)$. Since, $\left(\left\|t_{\text {max }}\right\|-\left\|t_{\text {min }}\right\|\right)-\left(\left\|t_{2}\right\|-\left\|t_{1}\right\|\right) \geq 0$ is implied by $I_{\text {arc }}^{\text {callset }}$, we map all $\operatorname{arc} / 2$ atoms in the call set, to $\mathbb{N}$-closed polynomials.

To obtain an integrated approach, incorporating invariance and termination conditions, we introduce a symbolic form for level mappings, parameterizable by invariance relations. For $\operatorname{arc} / 2$ atoms, we have $\left|\operatorname{arc}\left(t_{1}, t_{2}\right)\right|=i i_{0}+i i_{1} .\left\|t_{1}\right\|+$ $i i_{2} .\left\|t_{2}\right\|-\left(i o_{0}+i o_{1} \cdot\left\|t_{1}\right\|+i o_{2} .\left\|t_{2}\right\|\right)$ as its symbolic form, which depends on the invariance relation holding on $\operatorname{arc} / 2$ atoms in the call set. Thus, $\forall t_{1}, t_{2} \in \operatorname{Term}_{P}$ :

$$
\begin{gathered}
i i_{0}^{1}+i i_{1}^{1} \cdot\left\|t_{1}\right\|+i i_{2}^{1} \cdot\left\|t_{2}\right\| \geq i o_{0}^{1}+i o_{1}^{1} \cdot\left\|t_{1}\right\|+i o_{2}^{1} \cdot\left\|t_{2}\right\| \wedge i i_{0}^{2}+i i_{1}^{2} \cdot\left\|t_{1}\right\|+i i_{2}^{2} \cdot\left\|t_{2}\right\| \geq \\
i o_{0}^{2}+i o_{1}^{2} \cdot\left\|t_{1}\right\|+i o_{2}^{2} \cdot\left\|t_{2}\right\| \wedge i i_{0}^{3}+i i_{1}^{3} \cdot\left\|t_{1}\right\|+i i_{2}^{3} \cdot\left\|t_{2}\right\| \geq i o_{0}^{3}+i o_{1}^{3} \cdot\left\|t_{1}\right\|+i o_{2}^{3} \cdot\left\|t_{2}\right\| \rightarrow \\
i i_{0}+i i_{1} \cdot\left\|t_{1}\right\|+i i_{2} \cdot\left\|t_{3}\right\| \geq i o_{0}+i o_{1} \cdot\left\|t_{1}\right\|+i o_{2} \cdot\left\|t_{3}\right\|
\end{gathered}
$$

Notice that the symbolic form, used in existing constraint-based approaches [5], is implied by default. After all, $i i_{0}+i i_{1} \cdot\left\|t_{1}\right\|+i i_{2} .\left\|t_{2}\right\| \geq 0$ is trivially implied, since only polynomials are constructed with positive integer coefficients. Therefore, if termination can be proven by existing systems, we can prove it as well.

As termination conditions, we obtain now:

$$
\begin{gathered}
\forall t_{1}, t_{2}, t_{3}:\left(t_{1}, t_{2}\right) \in I_{\text {arc callet }}^{\text {cals }} \wedge\left(t_{2}, t_{3}\right) \in I_{\text {arc }}^{\text {callset }} \rightarrow \\
i i_{0}+i i_{1} \cdot\left\|t_{1}\right\|+i i_{2} \cdot\left\|t_{2}\right\|-\left(i o_{0}+i o_{1} \cdot\left\|t_{1}\right\|+i o_{2} \cdot\left\|t_{2}\right\|\right) \geq i i_{0}+i i_{1} \cdot\left\|t_{1}\right\|+i i_{2} \cdot\left\|t_{3}\right\|-\left(i o_{0}+i o_{1} \cdot\left\|t_{1}\right\|+i o_{2} \cdot\left\|t_{3}\right\|\right) \\
\forall t_{1}, t_{2}, t_{3}:\left(t_{1}, t_{2}\right) \in I_{\text {arc }}^{\text {calset }} \wedge\left(t_{2}, t_{3}\right) \in I_{\text {arc }}^{\text {callset }} \rightarrow \\
i i_{0}+i i_{1} \cdot\left\|t_{2}\right\|+i i_{2} \cdot\left\|t_{3}\right\|-\left(i o_{0}+i o_{1} \cdot\left\|t_{2}\right\|+i o_{2} \cdot\left\|t_{3}\right\|\right) \geq i i_{0}+i i_{1} \cdot\left\|t_{1}\right\|+i i_{2} \cdot\left\|t_{3}\right\|-\left(i o_{0}+i o_{1} \cdot\left\|t_{1}\right\|+i o_{2} \cdot\left\|t_{3}\right\|\right)
\end{gathered}
$$

Solving these conditions using the techniques in [5], provides us with the aforementioned interpretation for arc/2 constraints, proving termination.

Our technique is also applicable to LP. Similarly, invariance relations for atoms used in calls, can be derived on the basis of an invariance relation for the query atoms. We demonstrate this on the following LP program.

$$
a(\operatorname{Max}, \operatorname{Max}) . \quad a(N, \operatorname{Max}):-\operatorname{neq}(N, \operatorname{Max}), a(s(N), \operatorname{Max}) .
$$

Currently, no technique for termination analysis of LP can handle such programs. Since a call to $n e q / 2$ also succeeds whenever the first term of an $a / 2$ atom is bigger than the second, the program will run forever, as for such queries the first argument has no upper bound. Thus, in order to prove termination, we have to consider that a query consists of $a\left(t_{1}, t_{2}\right)$ atoms, where $t_{1} \prec t_{2}$, represented using an invariance relation, $I_{a / 2}^{\text {query }}=\left\{\left(t_{1}, t_{2}\right) \mid t_{1}, t_{2} \in\right.$ Term $\left._{P},\left\|t_{2}\right\|>\left\|t_{1}\right\|\right\}$. 
Consequently, to derive $I_{a / 2}^{\text {callset }}$, we initially set it to a general symbolic form, $\left\{\left(t_{1}, t_{2}\right) \mid t_{1}, t_{2} \in \operatorname{Term}_{P} \wedge i i_{0}^{1}+i i_{1}^{1} \cdot\left\|t_{1}\right\|+i i_{2}^{1} \cdot\left\|t_{2}\right\| \geq i o_{0}^{1}+i o_{1}^{1} \cdot\left\|t_{1}\right\|+i o_{2}^{1} \cdot\left\|t_{2}\right\|\right\}$ and then, we formulate invariance conditions. To express $I_{a / 2}^{\text {query }} \subseteq I_{a / 2}^{\text {callset }}$, we get that $\forall t_{1}, t_{2} \in \operatorname{Term}_{P}:\left\|t_{2}\right\|>\left\|t_{1}\right\| \rightarrow i i_{0}^{1}+i i_{1}^{1} \cdot\left\|t_{1}\right\|+i i_{2}^{1} \cdot\left\|t_{2}\right\| \geq i o_{0}^{1}+$ $i o_{1}^{1} \cdot\left\|t_{1}\right\|+i o_{2}^{1} \cdot\left\|t_{2}\right\|$. For the clause, we obtain that $\forall t_{1}, t_{2} \in \operatorname{Term}_{P}: i i_{0}^{1}+i i_{1}^{1} \cdot\left\|t_{1}\right\|+$ $i i_{2}^{1} .\left\|t_{2}\right\| \geq i o_{0}^{1}+i o_{1}^{1} \cdot\left\|t_{1}\right\|+i o_{2}^{1} \cdot\left\|t_{2}\right\| \wedge\left(\left\|t_{1}\right\|>\left\|t_{2}\right\| \vee\left\|t_{2}\right\|>\left\|t_{1}\right\|\right) \rightarrow i i_{0}^{1}+$ $i i_{1}^{1} \cdot\left\|s\left(t_{1}\right)\right\|+i i_{2}^{1} \cdot\left\|t_{2}\right\| \geq i o_{0}^{1}+i o_{1}^{1} \cdot\left\|s\left(t_{1}\right)\right\|+i o_{2}^{1} \cdot\left\|t_{2}\right\|$. Here, $R_{n e q / 2}=\left\{\left(t_{1}, t_{2}\right) \mid\right.$ $\left.\left\|t_{1}\right\|>\left\|t_{2}\right\| \vee\left\|t_{2}\right\|>\left\|t_{1}\right\|\right\}$ estimates the effect of a call to neq/2. Then, a suitable polynomial level mapping for $a / 2$ atoms is derived on the basis of $I_{a / 2}^{\text {callset }}$. That is, $\forall t_{1}, t_{2} \in \operatorname{Term}_{P}: i i_{0}^{1}+i i_{1}^{1} \cdot\left\|t_{1}\right\|+i i_{2}^{1} \cdot\left\|t_{2}\right\| \geq i o_{0}^{1}+i o_{1}^{1} \cdot\left\|t_{1}\right\|+i o_{2}^{1} \cdot\left\|t_{2}\right\| \rightarrow$ $i i_{0}+i i_{1} \cdot\left\|t_{1}\right\|+i i_{2} \cdot\left\|t_{2}\right\| \geq i o_{0}+i o_{1} \cdot\left\|t_{1}\right\|+i o_{2} \cdot\left\|t_{2}\right\|$.

To prove termination, we must show that all recursive body atoms are smaller than the atom used in the head [2]. This results for the LP program, in the following condition: $\forall t_{1}, t_{2} \in \operatorname{Term}_{P}: i i_{0}^{1}+i i_{1}^{1} \cdot\left\|t_{1}\right\|+i i_{2}^{1} \cdot\left\|t_{2}\right\| \geq i o_{0}^{1}+i o_{1}^{1} \cdot\left\|t_{1}\right\|+$ $i o_{2}^{1} \cdot\left\|t_{2}\right\| \wedge\left(\left\|t_{1}\right\|>\left\|t_{2}\right\| \vee\left\|t_{2}\right\|>\left\|t_{1}\right\|\right) \rightarrow i i_{0}+i i_{1} \cdot\left\|t_{1}\right\|+i i_{2} \cdot\left\|t_{2}\right\|-\left(i o_{0}+i o_{1} \cdot\left\|t_{1}\right\|+\right.$ $\left.i o_{2} .\left\|t_{2}\right\|\right) \geq i i_{0}+i i_{1} \cdot\left\|s\left(t_{1}\right)\right\|+i i_{2} \cdot\left\|t_{2}\right\|-\left(i o_{0}+i o_{1} \cdot\left\|s\left(t_{1}\right)\right\|+i o_{2} \cdot\left\|t_{2}\right\|\right)$. When solving the termination, invariance and interargument conditions, we obtain $\left|a\left(t_{1}, t_{2}\right)\right|=$ $\left\|t_{2}\right\|-\left\|t_{1}\right\|$ as one possible level mapping for $a / 2$ atoms, proving termination.

To conclude, we proposed a new constraint-based approach, more powerful than existing approaches in LP. Our approach can handle both LP and CHR programs containing bounded increase, integer arithmetic or programs that only terminate for subsets of considered queries. We overcame these problems by introducing invariance relations, resulting in a useful specification for relations holding among the instantiated parts of call atoms and thus complementing call types. As such, we are able to represent more restricted kinds of queries, related to the use of domains or even global properties of queries, such as cycle-freeness of a query graph. In the continuation of this work, we will formalize this technique and further evaluate it, in the context of CHR and Logic Programs.

\section{References}

1. A. Bossi, N. Cocco, and M. Fabris. Proving termination of logic programs by exploiting term properties. TAPSOFT91, p.153-180 (1991).

2. D. De Schreye and S. Decorte. Termination of logic programs: the never-ending story. JLP, vol.19-20, p.199-260 (1994).

3. T. Frühwirth. Theory and practice of constraint handling rules. JLP, vol.37(1-3), p.95-138 (1998).

4. G. Janssens and M. Bruynooghe. Deriving descriptions of possible values of program variables by means of abstract interpretation. JLP, vol.13(2-3), p.205-258 (1992).

5. M.T. Nguyen and De Schreye. Polynomial interpretations as a basis for termination analysis of logic programs. In ICLP 2005, p.311-325 (2005).

6. P. Pilozzi and D. De Schreye. Termination analysis of chr revisited. In ICLP08, p.501-515 (2008).

7. T. Schrijvers. Analyses, optimizations and extensions of constraint handling rules: Ph.d. summary. In ICLP05, p.435-436 (2005). 